\title{
SCIWORA/SCIWORET traumático: experiencia de tres años en un hospital especializado
}

\section{Traumatic SCIWORA/SCIWORET: Three years experience in a specialized hospital}

\author{
Arturo García-Galicia ${ }^{1 *}$, Edgar Corpus-Mariscal2 ${ }^{2}$, José J. Torreblanca-Reyes ${ }^{1}$, Luis F. González-Ramos², \\ Rodolfo G. Barragán-Hervella², Álvaro J. Montiel-Jarquín ${ }^{1}$ y Carlos F. Morales-Flores² \\ ${ }^{1}$ Unidad Médica de Alta Especialidad, Hospital de Especialidades de Puebla; 2 Unidad Médica de Alta Especialidad, Hospital de Traumatología y \\ Ortopedia. Centro Médico Nacional "General de División Manuel Ávila Camacho", Instituto Mexicano del Seguro Social, Puebla, México
}

\begin{abstract}
Resumen
Introducción: La SCIWORA (spinal cord injury without radiographic abnormality) es una lesión medular espinal con radiología normal en pacientes con déficit neurológico de origen medular. El acrónimo SCIWORET (spinal cord injury without radiologic evidence of trauma) se emplea en adultos, quienes suelen presentar cambios espondilíticos. Son lesiones devastadoras económica, psicológica y socialmente. Objetivo: Describir la evolución neurológica y calidad de vida de los pacientes con SCIWORA/SCIWORET durante el periodo 2016-2018 en un hospital de 3.er nivel de atención. Material y métodos: Estudio descriptivo, longitudinal, unicéntrico, con seguimiento a pacientes con SCIWORA/SCIWORET, de 5-75 años, durante 2016-2018. Se excluyeron pacientes con traumatismo craneoencefálico moderado-grave. Se analizó la evolución neurológica y la calidad de vida mediante las escalas ASIA (American Spinal Injury Association) y EQ-5D (European Quality of Life-5 Dimensions), respectivamente. Se utilizó estadística descriptiva. Resultados: 14 pacientes reclutados, 9 (64\%) fueron hombres, edades 7-66 años (media 36.3). La cinemática más frecuente: caída de propia altura (6 pacientes); 9 pacientes calificaron ASIA D, 3 ASIA C y 2 ASIA B. Nueve mejoraron al menos un nivel ASIA a seis meses postrauma. Doce se reintegraron a sus actividades cotidianas y dos presentaron secuelas neurológicas permanentes. Conclusión: La mayoría de los pacientes con SCIWORA/SCIWORET tratados conservadoramente recuperaron al menos un nivel ASIA y registraron buena calidad de vida a seis meses tras la lesión.
\end{abstract}

Palabras clave: SCIWORA. SCIWORET. Lesión medular. Evolución neurológica. Calidad de vida.

\section{Abstract}

Background: SCIWORA (Spinal Cord Injury Without Radiographic Abnormality) refers to spinal cord injury with normal radiology in patients with neurological deficit from spinal origin. SCIWORET (Spinal Cord Injury Without Radiologic Evidence of Trauma) is used in adults, which present spondilitic changes. Both are economically, psychologically and socially devastating injuries. Aim: To describe neurologic evolution in patients with SCIWORA/SCIWORET diagnosis during 2016-2018 at a $3^{\text {rd }}$ attention level. Methods: Descriptive, longitudinal, unicentric study, following patients with

Correspondencia:

*Arturo García-Galicia

E-mail: arturo.garciaga@imss.gob.mx
Disponible en internet: 05-05-2021 Rev Hosp Jua Mex. 2021;88(2):50-55

www.revistahospitaljuarez.com 1405-9622/○ 2020 Sociedad Médico-Quirúrgica del Hospital Juárez de México, A.C. Publicado por Permanyer. Este es un artículo open access bajo la licencia CC BY-NC-ND (http://creativecommons.org/licenses/by-nc-nd/4.0/). 
SCIWORA/SCIWORET diagnosis, 5-75 years-old (yo), during 2016-2018. Patients with moderate-severe craniocerebral trauma were excluded. Neurological evolution and health related quality of life (HRQOL) were measured with ASIA and EQ-5D scales respectively. Descriptive statistics were used. Results: 14 patients recruited, 9 (64\%) men, 7-66 yo (media 36.3). More frequent trauma mechanism: fall from standing height (6 patients); 9 patients were ASIA D, 3 ASIA C, 2 ASIA B. Nine patients got better at least 1 ASIA level at 6 months post- trauma. Twelve patients got back to usual activities, 2 presented permanent neurological aftermaths. Conclusion: Most of patients with SCIWORA/SCIWORET diagnosis treated with conservative management recovered at least 1 ASIA level, and registered good HRQOL at 6 months after trauma.

Key words: SCIWORA. SCIWORET. Spinal cord injury. Neurological evolution. Health related quality of life.

\section{Introducción}

La Organización Mundial de la Salud (OMS) define la lesión medular como todo daño en la médula espinal ocasionado por traumatismos (accidente automovilístico, caídas, actos de violencia), enfermedad (mielitis transversa) o cáncer, en orden de frecuencia. Se calcula una incidencia mundial de $40-80$ casos por millón de habitantes anualmente. Los pacientes con esta afección son de dos a cinco veces más propensos a morir prematuramente que los que no la padecen $^{1-4}$.

La lesión medular varía entre niños y adultos: la columna cervical pediátrica es hipermóvil, debido a la laxitud ligamentaria y a la horizontalización de las facetas vertebrales. Quizá por ello es que en niños se han encontrado con mayor frecuencia lesiones medulares $\sin$ anomalías radiográficas ${ }^{5,6}$. Para estos casos se acuñó el término SCIWORA (spinal cord injury without radiographic abnormality), definida como una lesión medular espinal con radiología normal en pacientes con déficit neurológico de origen medular. Su etiología permanece poco clara. En los adultos se utiliza el acrónimo SCIWORET (spinal cord injury without radiologic evidence of trauma), en ellos la lesión medular espinal suele presentar cambios espondilíticos ${ }^{1-4}$.

Estas patologías representan un importante problema de salud debido a las implicaciones psicológicas, sociales y económicas para el paciente, para su familia y para los sistemas de salud, y requieren un tratamiento integral por sus secuelas y complicaciones ${ }^{2-7}$.

El cuadro neurológico más frecuente es el síndrome medular central, principalmente en casos de trauma de baja energía (43\%), accidentes automovilísticos (33\%) y caída de altura $(12 \%)^{2}$.

Los pacientes menores de 8 años y los mayores de 60 son los más propensos a esta patología, debido a su constitución anatómica y biomecánica, aunque esta condición se puede presentar en cualquier época de la vida $^{1-3}$.
La llegada de la resonancia magnética (RM) nos permite demostrar lesiones en la médula en aquellos pacientes con radiología simple $(\mathrm{Rx})$ y tomografía computarizada (TC) normales, al detectar lesiones osteoligamentarias o medulares que pasaban inadvertidas. Estos casos dejan de considerarse verdaderas SCIWORA y representan un $90 \%$ del total. El 10\% restante sí presentan Rx, TC y RM normales $2,4,8-12$.

Dentro de las patologías detectables solo por RM se encuentran las lesiones extramedulares (hernia de disco, estenosis de canal, lesión de ligamento vertebral común anterior o complejo ligamentario posterior y hematoma intracanal) e intramedulares (edema, contusión y hemorragia) $)^{8,9}$. Su relevancia puede ser tan significativa como para determinar el pronóstico del paciente 8 ,13-17.

La RM no se encuentra disponible en todos los centros de trauma y/o no se realiza de primera intención, por lo que la SCIWORA sigue siendo una entidad sobrediagnosticada.

La medición de la calidad de vida relacionada con la salud (CVRS) es muy útil para estudiar la salud de la población y analizar el costo-efectividad de las intervenciones sanitarias. En el presente estudio se eligió el EUROQOL-5D (EQ-5D, European Quality of Life-5 Dimensions), un instrumento genérico para población general y pacientes con diferentes patologías. En él, el propio individuo valora su estado de salud, primero en cinco dimensiones con tres valores de gravedad cada uno (sistema descriptivo), y luego en una escala visual analógica (EVA), más general, obteniéndose un «estado de salud». Un tercer elemento es el índice de valores que se obtiene para cada estado de salud. Este índice oscila entre el valor 1 (el mejor estado de salud) y el 0 (la muerte) ${ }^{18}$.

El objetivo general fue describir la evolución neurológica y la calidad de vida de los pacientes con diagnóstico de SCIWORA y SCIWORET tratados en el Hospital de Traumatología y Ortopedia de Puebla de 2016 a 2018. 
Tabla 1. Características de los pacientes atendidos en 2016-2018 con diagnóstico de SCIWORA/SCIWORET (spinal cord injury without radiographic abnormality/spinal cord injury without radiologic evidence of trauma) en la Unidad Médica de Alta Especialidad Hospital de Traumatología y Ortopedia del Centro Médico Nacional “General de División Manuel Ávila Camacho", del Instituto Mexicano del Seguro Social

\begin{tabular}{|c|c|c|c|c|c|c|}
\hline Edad & \multicolumn{2}{|c|}{$\begin{array}{c}\text { Media } \\
36.3 \text { años }\end{array}$} & $\begin{array}{l}\text { Mínimo } \\
7 \text { años }\end{array}$ & \multicolumn{2}{|c|}{$\begin{array}{l}\text { Máximo } \\
66 \text { años }\end{array}$} & $\begin{array}{c}\mathrm{DE} \\
18.595 \text { años }\end{array}$ \\
\hline Sexo & \multicolumn{3}{|c|}{$\begin{array}{l}\text { Femenino } \\
9 \text { años }\end{array}$} & \multicolumn{3}{|c|}{$\begin{array}{l}\text { Masculino } \\
5 \text { años }\end{array}$} \\
\hline $\begin{array}{l}\text { Antecedentes } \\
\text { patológicos }\end{array}$ & \multicolumn{2}{|c|}{$\begin{array}{c}\text { Sin antecedentes } \\
9\end{array}$} & $\begin{array}{c}\text { HTA } \\
2\end{array}$ & $\begin{array}{c}\mathrm{HTA}+\mathrm{DM} \\
2\end{array}$ & \multicolumn{2}{|c|}{$\begin{array}{c}\mathrm{HTA}+\mathrm{IVC} \\
1\end{array}$} \\
\hline \multirow{3}{*}{$\begin{array}{l}\text { Mecanismo del } \\
\text { trauma y } \\
\text { afectación } \\
\text { neurológica }\end{array}$} & $\begin{array}{l}\text { Caída de su } \\
\text { propia altura }\end{array}$ & $\begin{array}{l}\text { Caída de } \\
\text { mayor altura }\end{array}$ & $\begin{array}{l}\text { Levantamiento } \\
\text { de objeto pesado }\end{array}$ & $\begin{array}{l}\text { Contusión directa } \\
\text { toracolumbar }\end{array}$ & $\begin{array}{l}\text { Accidente } \\
\text { automovilístico }\end{array}$ & Actividades deportivas \\
\hline & 6 pacientes & 2 pacientes & 2 pacientes & 2 pacientes & 1 paciente & 1 paciente \\
\hline & $\begin{array}{l}5 \text { central, } \\
1 \text { anterior }\end{array}$ & Central & Central & $\begin{array}{l}1 \text { anterior, } 1 \\
\text { posterior }\end{array}$ & Central & Central \\
\hline $\begin{array}{l}\text { Estancia } \\
\text { hospitalaria }\end{array}$ & \multicolumn{2}{|c|}{$\begin{array}{c}\text { Menos de } 3 \text { días } \\
4\end{array}$} & \multicolumn{2}{|c|}{$\begin{array}{c}\text { 3-7 días } \\
7\end{array}$} & \multicolumn{2}{|c|}{$\begin{array}{c}\text { Más de } 7 \text { días } \\
3\end{array}$} \\
\hline Complicaciones & \multicolumn{2}{|c|}{$\begin{array}{c}\text { Sin complicaciones } \\
11\end{array}$} & \multicolumn{2}{|c|}{$\begin{array}{l}\text { Infección urinaria } \\
2\end{array}$} & \multicolumn{2}{|c|}{$\begin{array}{c}\text { Cetoacidosis diabética } \\
1\end{array}$} \\
\hline
\end{tabular}

DE: desviación estándar; HTA: hipertensión arterial; DM: diabetes mellitus; IVC: insuficiencia venosa crónica.

\section{Métodos}

Se realizó un estudio descriptivo, transversal, en el Hospital de Traumatología y Ortopedia de Puebla del Instituto Mexicano del Seguro Social. Fue aprobado por el Comité Local de Investigación y Ética en Investigación en Salud n. ${ }^{\circ} 2105$ (registro: R-2017-2105-17). Se incluyeron pacientes atendidos de 2016 a 2018, de ambos sexos, con diagnóstico de SCIWORA/SCIWORET, de 5 a 75 años de edad, que firmaron consentimiento informado (en los menores de 18 años los padres firmaron el consentimiento). Se excluyeron pacientes con diagnóstico de traumatismo craneoencefálico (TCE) moderado o grave, aquellos con síntomas neurológicos previos al trauma y los que no aceptaron (o cuyos padres no aceptaron) entrar al estudio.

Los pacientes se captaron en el área de urgencias y en el módulo de columna de la unidad. Se utilizaron radiografías simples en proyección anteroposterior y lateral de columna de la región afectada. Posteriormente se realizó TC simple de la misma zona afectada, y si se reportaba sin evidencia de lesión, se realizaba RM.

A su llegada al hospital y/o establecido el diagnóstico, a todos los pacientes se les aplicó inmovilización simple, y se administró metilprednisolona a dosis habituales ${ }^{4}$.

Se les instaló una vía venosa periférica y se administraron analgésicos intravenosos, soluciones isotónicas y gastroprotectores (inhibidores de la bomba de protones).

Los pacientes fueron evaluados con la escala ASIA (American Spinal Injury Association) a su llegada a la unidad y a los seis meses de seguimiento, y con EQ-5D a los seis meses de seguimiento.

Se utilizó estadística descriptiva.

\section{Resultados}

Se reclutaron 14 pacientes en total con diagnóstico de SCIWORA Y SCIWORET. Nueve (64\%) fueron hombres y cinco (36\%) mujeres. Cuatro fueron menores de edad y diez mayores de 18 años. Las principales características se muestran en la tabla 1. No se eliminó ningún paciente.

El trauma de baja energía reportó la mayor frecuencia. En menores de 18 años los eventos traumáticos correspondieron a un accidente automovilístico, una caída de azotea, una lesión deportiva y una caída de escaleras. Todos presentaron síndrome medular incompleto (18) y el cuadro neurológico que prevaleció en estos casos fue el síndrome medular central en 11 pacientes (78.5\%). La estancia hospitalaria fue mayoritariamente de 3-7 días.

Las complicaciones reportadas fueron escasas (solo en tres pacientes), dos reportaron infección en vías 
urinarias y uno cetoacidosis diabética. Todos se recuperaron satisfactoriamente de las complicaciones.

Por lo que respecta a la evolución clínica, se muestra la calificación de la CVRS (con EQ-5D) a los seis meses en la figura 1. Es de destacar que si bien seis pacientes se reportaron sin limitaciones, uno reportó afectación en todas las dimensiones del instrumento además de depresión moderada y uno más reportó postración en cama; 12 pacientes se reintegraron a sus actividades laborales y cotidianas. La aplicación del EQ-5D para CVRS resultó como sigue: para los seis pacientes sin limitaciones, puntuación de 1; para los que reportaron problemas en actividades cotidianas el índice punteó en 0.7947 , para los que reportaron dolor moderado fue de 0.7902 , para el paciente con afectación en todos los dominios y con depresión, fue de 0.2215; y para el paciente postrado en cama fue de 0.1363 . Entre paréntesis se muestran los valores del índice de CVRS del EQ-5D. Los pacientes referidos con "depresión moderada» y «postración en cama» reportaron afectación en todos los dominios.

En cuanto a la evaluación con la escala ASIA al ingreso de los pacientes, la más frecuente fue la clase $\mathrm{D}, \mathrm{y}$ a los seis meses las clases D y E (Tabla 2); nueve pacientes $(64.3 \%)$ reportaron mejoría un nivel de la escala, y uno $(7.1 \%)$ la reportó en dos niveles, la frecuencia en la mejoría se muestra en la figura 2.

\section{Discusión}

Para el diagnóstico de los casos verdaderos de SCIWORA, la evaluación radiológica de la lesión medular requiere una RM normal4-13,19-22. En el presente trabajo todos los pacientes fueron diagnosticados por RM.

El sexo más frecuente fue el masculino, semejante a lo que menciona la OMS y Parente en el 2005, este último en niños ${ }^{3,23}$.

La posibilidad de recuperación neurológica de estos pacientes está relacionada en primera instancia con la lesión inicial, el diámetro del canal, la edad del paciente, el tamaño de la lesión y la severidad del cuadro neurológico; es por eso que la cirugía no es el estándar de oro para el tratamiento 3,13,19,20,24,25. Particularmente en los casos de SCIWORET, el déficit neurológico no presenta lesiones radiográficas traumáticas. Los cambios degenerativos propios del adulto (como la degeneración espondiloartrósica) producen una excesiva tracción de la médula espinal durante el accidente, con mecanismo principal la hiperextensión $^{4-9,20}$. El resultado es una descompensación de la

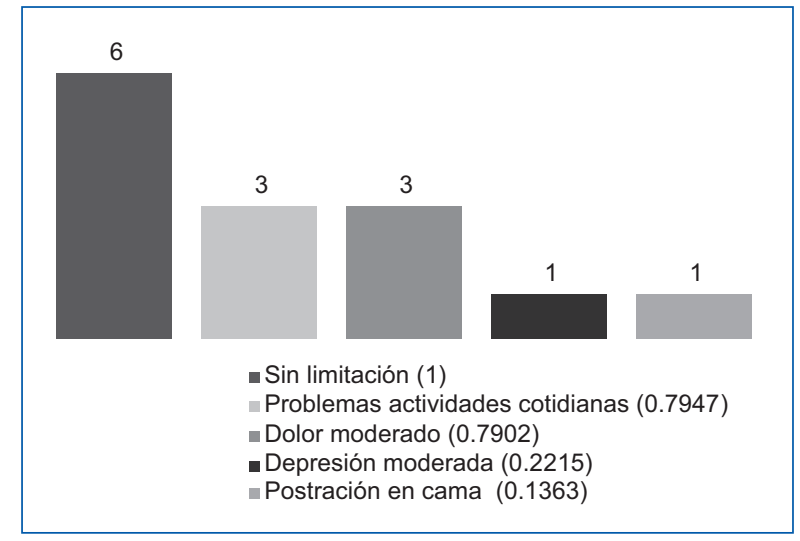

Figura 1. Calidad de vida a los seis meses con EUROQ0L-5D. Entre paréntesis se muestran los valores del índice de calidad de vida relacionada con la salud. Los pacientes referidos con "depresión moderada" y "postración en cama" reportaron afectación en todos los dominios.

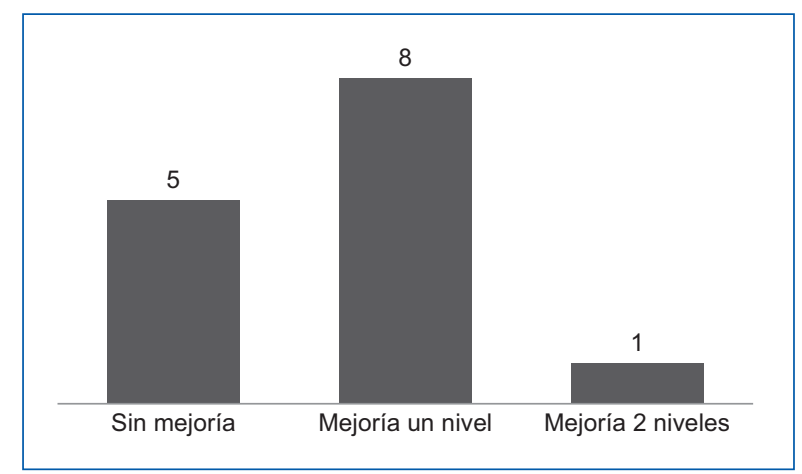

Figura 2. Mejoría en la escala de ASIA (American Spinal Injury Association) a los seis meses de seguimiento.

Tabla 2. Evaluación de los pacientes con escala de ASIA (American Spinal Injury Association) al ingreso y a los seis meses

\begin{tabular}{|l|l|l|l|l|l|}
\hline \multicolumn{7}{|c|}{ Evaluación ASIA } \\
\hline & A & B & C & D & E \\
\hline Al ingreso & 0 & 2 & 3 & 9 & 0 \\
\hline A los 6 meses & 0 & 1 & 1 & 6 & 6 \\
\hline
\end{tabular}

relación continente/contenido en columnas artrósicas asintomáticas, pudiendo ser devastador para el paciente $^{4,9,12,20}$.

Se ha utilizado el tratamiento con esteroides en esquemas ampliamente difundidos, aspecto en controversia reciente cada vez mayor, con tendencia a no 
aconsejarlo salvo en casos extremos ${ }^{4,25}$. Para Saruhashi, et al., aquellos que tenían buena respuesta al tratamiento con corticosteroides eran manejados con inmovilización y los que presentaban cuadros severos o progresivos deberían ser intervenidos quirúrgicamente ${ }^{8}$. En el presente trabajo, todos los pacientes respondieron adecuadamente al tratamiento conservador, que incluyó esteroides. Se encuentra en marcha una readecuación de los esquemas de tratamiento en la Unidad.

Existen reportes acerca del origen de la lesión medular. Mientras que algunos autores refieren mayor frecuencia de los traumatismos en pacientes pediátricos, en otros estudios predominan las causas no traumáti$\mathrm{cas}^{3,4}$. Estas divergencias parecen deberse a las unidades médicas de origen de los autores. El presente estudio se realizó en pacientes de una unidad de 3 .er nivel de atención en traumatología y ortopedia, y fueron tratados conservadoramente. Prevalecieron los mayores de 18 años, la etiología traumática y el trauma de baja energía (incluyendo caída de su propia altura), también la calificación ASIA D y la evolución neurológica favorable en la mayoría de los pacientes, muy parecido a lo encontrado en otros estudios de similares $^{4,9,13}$.

El seguimiento en nuestros pacientes llegó hasta los seis meses, final de la fase intermedia (dos semanas-seis meses) e inicio de la tardía (seis meses-dos años), y quizá por eso los resultados de la evolución y la calidad de vida sean más bien halagüeños. En la fase tardía continúa la cicatrización glial y ocurre la maduración completa de la lesión hasta los dos años. Ello probablemente mejoraría aún más la sintomatología, pero, por otro lado, se llega a reportar hasta un $30 \%$ de disfunción neurológica tardía. De cualquier manera, un seguimiento más prolongado sería ilustrador 20,23,26-30.

Los resultados del presente estudio permiten afirmar que, en concordancia con los reportes internacionales, la mayoría de los pacientes con diagnóstico de SCIWORA/SCIWORET tratados de forma conservadora ganaron por lo menos un nivel de ASIA y registraron buena calidad de vida a los seis meses de ocurrida la lesión.

\section{Conflicto de intereses}

Los autores declaran no tener conflicto de intereses alguno.

\section{Financiamiento}

La presente investigación no ha recibido ninguna beca específica de agencias de los sectores público, comercial o sin ánimo de lucro.

\section{Responsabilidades éticas}

Protección de personas y animales. Los autores declaran que para esta investigación no se han realizado experimentos en seres humanos ni en animales.

Confidencialidad de los datos. Los autores declaran que han seguido los protocolos de su centro de trabajo sobre la publicación de datos de pacientes.

Derecho a la privacidad y consentimiento informado. Los autores han obtenido el consentimiento informado de los pacientes y/o sujetos referidos en el artículo.

\section{Bibliografía}

1. Sesmat $H$, Hayoun $T$, Bonnet JP, Poli-Mérol ML, Bourelle S. Spinal cord injury without radiographic abnormality (SCIWORA): About 3 cases and review of the literature. Biomed J Sci\&Tech Res [Internet]. 2018;8:620811 [consultado: 23 de septiembre de 2019]. Disponible en: https://biomedres.us/fulltexts/BJSTR.MS.ID.001584.php

2. Aso-Escario J, Sebastián-Sebastián C, Aso-Vizán A, Martínez-Quiñones JV, Consolini F, Arregui-Calvo R. Lesión medular con anormalidad radiológica. Etiología, diagnóstico y problemática médico-legal. Rev Esp Med Legal. 2017;209:1-7.

3. Mancilla-Ramírez A, García-Miranda GA. Frecuencia de la lesión medular pediátrica en un centro de rehabilitación. Experiencia de nueve años. Rev Mex Neuroci. 2018;19:36-44

4. Atesok K, Tanaka N, O'Brien A, Robinson Y, Pang D, Deinlein D, et al. Posttraumatic spinal cord injury without radiographic abnormality. Adv Orthop. 2018;2018:7060654

5. Brague D, Plas B, Vinchon M, Charni S, DiRocco F, Sacko O, et al. Multicenter study of 37 pediatric patients with SCIWORA or other spinal cord injury without associated bone lesión. Orthop Traumatol Surg Res. 2019;106(1):167-71.

6. Bansal ML, Sharawat R, Mahajan R, Dawar H, Mohapatra B, Das K, et al. Spinal injury in Indian children: Review of 204 cases. Global Spine J. 2020;10(8):1034-9.

7. Brenes-Rojas Y. Lesiones medulkares agudas. Rev Med CR Centroam. 2016;73(620):645-52.

8. Hendey GW, Wolfson AB, Mower WR, Hoffman JR; National Emergency X-Radiography Utilization Study Group. Spinal cord injury without radiographic abnormality: results of the National Emergency $\mathrm{X}$-Radiography Utilization Study in blunt cervical trauma. J Trauma. 2002;53:1-4

9. Bazán PL. Trascendencia del cuadro SCIWORA en adultos. Coluna/ Columna. 2015;14:134-7

10. Yucesoy K, Yuksel KZ. SCIWORA in MRI era. Clin Neurol Neurosurg. 2008;110:429-33.

11. Khan AA, Mahmood S, Saif T, Gul A. Spinal cord injury without radiographic abnormality (SCIWORA) in adults: A report of two cases. J Pak Med Assoc. 2017;67:1275-7.

12. Bonfanti L, Donelli V, Lunian M, Cerasti D, Cobianchi F, Cervellin G. Adult Spinal Cord Injury Without Radiographic Abnormality (SCIWORA). Two case reports and a narrative review. Acta Biomed. 2018;89:593-8.

13. Anglen J, Metzler M, Bunn P, Griffiths H. Flexion and extension views are not cost-effective in a cervical spine clearance protocol for obtunded trauma patients. J Trauma. 2002;52:54-9.

14. Asan Z. Spinal cord injury without radiological abnormality in adults: clinical and radiological discordance. World Neurosurg. 2018; 114:e1147-e1151.

15. Fischer I, Haas C, Raghupathi R, Jin Y. Spinal cord concussion: studying the potential risks of repetitive injury. Neural Regen Res. 2016;11:58-60. 
16. Jin Y, Bouyer J, Haas C, Fischer I. Evaluation of the anatomical and functional consequences of repetitive mild cervical contusion using a model of spinal concussion. Exp Neurol. 2015;271:175-88.

17. Jin Y, Bouyer J, Haas C, Fischer I. Behavioral and anatomical consequences of repetitive mild thoracic spinal cord contusion injury in the rat. Exp Neurol. 2014;257:57-69.

18. Herdman M, Badia X, Berra S. El EuroQol-5D: una alternativa sencilla para la medición de la calidad de vida relacionada con la salud en atención primaria. Aten Primaria. 2001;28:425-9.

19. Kunam VK, Velayudhan V, Chaudry ZA, Bobinski M, Smoker WRK, Reede DL. Incomplete cord síndromes: Clinical and imaging review. RadioGraphics. 2018;238:12201-2.

20. Baabor M, Cruz S, Villalón J. Actualization in pathophysiology and management of traumatic spinal cord injury. Literature review. Rev Chil Neurocirugia. 2016;42(2):144-50.

21. Dreizin D, Kim W, Kim JS, Boscak AR, Bodanapally UK, Munera F, et al. Will the real SCIWORA please stand up? Exploring clinicoradiologic mismatch in closed spinal cord injuries. AJR Am J Roentgenol. 2015;205:853-60.

22. Bozzo A, Marcoux J, Radhakrishna M, Pelletier J, Goulet B. The role of magnetic resonance imaging in the management of acute spinal cord injury. J Neurotrauma. 2011;28:1401-11.
23. Parente A, Navascués JA, Hernández E, Sánchez-París O, Cañizo A, Cerdá J, et al. Lesiones raquimedulares en la infancia. Cir Pediatr. 2005;18:132-5.

24. Yamazaki T, Yanaka K, Fujita K, Kamezaki T, Uemura K, Nose T. Traumatic central cord syndrome: analysis of factors affecting the outcome. Surg Neurol. 2005;63(2):95-9.

25. Qi C, Xia H, Miao D, Wang X, Li Z. The influence of timing surgery in the outcome of spinal cord injury without radiographic abnormality (SClWORA). J Orthop Surg Res. 2020;15(1):223.

26. Bracken MB, Shepard MJ, Collins WF, Holford TR, Young W, Baskin DS, et al. A randomized, controlled trial of methylprednisolone or naloxone in the treatment of acute spinal cord injury. Results of the second national acute spinal cord injury study. N Engl J Med. 1990;322:1405-11.

27. Kim DH, Ludwig SC, Vaccaro AR, Chang JC. Atlas de lesiones vertebrales en adultos y niños. Barcelona, España: Elsevier; 2010. pp. 11-20, 28-36.

28. Asan Z. Long term follow-up results of spinal concussion cases: Definition of late injuries of the spinal cord. World Neurosurg. 2018;120:e1325-e1330.

29. Nagoshi N, Tetreault L, Nakashima H, Nouri A, Fehlings MG. Return to play in athletes with spinal cord concussion: a systematic literature review. Spine J. 2017;17:291-302.

30. Tempel ZJ, Bost JW, Norwig JA, Maroon JC. Significance of T2 hyperintensity on 7 magnetic resonance imaging after cervical cord injury and return to play in professional 8 athletes. Neurosurgery. 2015;77(1):23-30. 